\title{
Evaluation of Safety of Pancreaticoduodenectomy for Patients with Benign Conditions
}

\author{
Bangren $X u^{1}$, Xiaowei Yuan ${ }^{2}$, Tiffany Wong ${ }^{1}, T T$ Cheung $^{3}, C M L^{3}$, Ji Ren ${ }^{1}$, Siyuan Qiu ${ }^{1}$, Chunhong Liu ${ }^{1}$ \\ and Hongtao $\mathrm{Zhu}^{1}$
}

${ }^{1}$ Department of Surgery, The University of Hong Kong-Shenzhen Hospital, Guangdong, China

${ }^{2}$ Department of Sugery, Guangzhou United Family Hospital, China

${ }^{3}$ Department of Surgery, The University of Hong Kong, Hong Kong, China

\begin{abstract}
Introduction: Pancreaticoduodenectomy (PD) is a complex and high-risk surgical procedure with substantial mortality and morbidity. Selected patients with benign conditions may need PD in case of ambiguous tissue diagnosis. There is limited data in the literature on the outcomes of patients with benign conditions undergoing PD. The purpose of this study is to determine the safety of PD in patients with benign conditions.
\end{abstract}

Materials \& Methods: From 2013 to 2018, 38 patients undergone pancreatectomy (PD [Group 1], $\mathrm{n}=9$; Distal pancreatectomy [Group 2], $n=29$ ) were retrospectively reviewed in a prospectively collected database. Short-term outcome in terms of procedure-related complication was evaluated.

Results: There was no significant difference in demographic features in both groups. Majority of patients was female (Group1: 66.6\%, Group 2: 75.8\%) and had no significant comorbidity (Group 1: 88.8\%, Group 2: 82.4\%). Patients in group 1 rarely presented with obstructive jaundice (median bilirubin level $=11.1 \mathrm{umol} / \mathrm{L}$ ). Disease spectrum in group 1 included insulinoma $(n=1)$, pancreatic tuberculosis $(n=1)$, Ig G4-related disease $(n=1)$, mucinous cystadenoma $(n=1)$, splenic aneurysm $(n=1)$, serous cystadenoma $(n=2)$ and benign neuroendocrine tumor $(n=2)$. Group 1 had significantly longer operation duration ( $470 \mathrm{~min}$ vs. $287 \mathrm{~min}, \mathrm{p}<0.001$ ), longer hospital stay ( 20 days vs. 14 days, $p=0.032$ ) and more interoperative blood loss $(600 \mathrm{ml}$ vs. $350 \mathrm{ml}, p=0.013$ ) than group 2. The postoperative pancreatic fistula rate of Group 1 was significantly lower than Group 2 (Grade A: $33.3 \%$ vs. $65.5 \%$; Grade B: $0 \%$ vs. $24.1 \%$; Grade C: $11.1 \%$ vs. $0 \%$ ), $(p=$ $0.004)$. There was no significant difference in other severe postoperative complications between the two groups. There was also no hospital mortality in both groups.

Conclusion: Pancreaticoduodenectomy can be safely performed for patients with benign diseases with postoperative pancreatic fistula rate.

\section{Keywords}

Benign conditions, Benign pancreatic tumor, Distal pancreatectomy, Pancreaticoduodenectomy, Evaluation of safety

\section{Introduction}

With the wide spread use of cross-sectional imaging examinations, more and more pancreatic benign tum or have been diagnosed. In patients with abdominal multi-slice spiral $\mathrm{CT}$ or MRI for reasons unrelated to pancreatic tumor, more than $2 \%$ of pancreatic tumor may be detected, which increases with age $[1,2]$. Benign pancreatic tumors are often difficult to diagnose and most are at risk of malignant transformation. Pancreaticoduodenectomy (PD) is the gold standard for benign and malignant tumors of the pancreatic head. PD is a multiple organ resection operation that requires removal of part of the stomach, extrahepatic bile ducts, and duodenum. However, one study found that $38 \%$ of patients suitable for resection of pancreatic cancer refused surgery [3]. Further- more, even after a complete resection, the 5-year survival rate is approximately 30 percent [4]. Rarely, benign conditions of the pancreatic head undergone PD to estimate safe.

The aims of the present study were to determine the safety of PD in patients with benign conditions. This data will

*Corresponding author: TT Cheung, Department of Surgery, The University of Hong Kong, Hong Kong, China

Accepted: October 09, 2020

Published online: October 11, 2020

Citation: Xu B, Yuan X, Wong T, et al. (2020) Evaluation of Safety of Pancreaticoduodenectomy for Patients with Benign Conditions. Clin Hematol Res 3(1):38-41 
Citation: Xu B, Yuan X, Wong T, et al. (2020) Evaluation of Safety of Pancreaticoduodenectomy for Patients with Benign Conditions. Clin Hematol Res 3(1):38-41

provide the foundation for clinical work to better guidance, and will ultimately contribute toward the establishment of an evidence-based management plan.

\section{Methods}

\section{Patients and data collection}

From January 2013 to June 2018, all adult patients followed in a Department of Hepatobiliary pancreas (University of Hongkong-Shenzhen Hospital, China), which is one of the largest pancreatic centers in China, The study were retrospectively screened. All patients older than 20 years who underwent a contrast abdominal computed tomography (CT) or an abdominal magnetic resonance imaging (MRI) for any reason were consecutively selected for the study. The patients with pancreatic head tumor underwent pancreaticoduodenectomy, and pathology showed benign tumor (eg: Insulinoma, mucinous cystic neoplasm, pancreatic tuberculosis, splenic aneurysm, serous cystadenoma, macrocystic serous cystadenoma, IgG4-related autoimmune pancreatitis). There were patients with distal pancreatic tumor underwent distal pancreatecto$\mathrm{my}$, and pathology showed benign tumor or malignancy.

\section{Statistical analysis}

Statistical Analysis IBM SPSS Statistical Software (version 24.0, IBM Corp, Armonk, NY, 2015) was used for all analysis. Categorical variables were compared using the Pearson's $\chi^{2}$ test, quantitative variables were compared among groups using the analysis of variance and Student $t$ test was used for compare unpaired data between 2 groups. Results are presented in percentage, as mean and SD or as median and interquartile range (IQR). All reported $P$ values are 2-tailed. $P$ values $\leq 0.05$ were considered as statistically significant. Variables were included if $P$ value was $\leq 0.1$ at univariate analysis. Well-known confounding factors (age, sex, and liver function) were also included in the models regardless of $P$ value at univariate analysis. Disease duration was not included to avoid overfitting and collinearity with age. The selected potential confounders were assessed in a Cox proportional hazards model. The study's observational measures were demographic characteristics, comorbidities, blood test (total bilirubin, albumin, CEA, CA199), intraoperative situation (operation duration, blood loss), pathology, length of stay, postoperative morbidities (pancreatic fistula, hydrothorax, ascites bile leakage, gastrointestinal bleeding, intra-abdominal bleeding, organ failure, ileus, gastric stasis), and impatient death. Classification of pancreatic fistula (PF) is basic on the ISGPF in 2016 [5]. The classification and grading of postoperative PFs are as follows: 1 . Biochemical Leak-Patients with elevated amylase but have no clinical symptoms or signs of local infection or sepsis. There is no deviation from the normal postoperative pathway and require no specific treatment such as antibiotics, total parenteral nutrition or somatostatin analogues. An operative drain may remain in place for up to three weeks. The patient is fed orally, remains clinically well and can adhere to an enhanced recovery pathway. 2 . Grade B-Patients have a clinical relevant sequela requiring change in management from the expected postoperative pathway such as pharmacologic treatment (eg, antibiotics, somatostatin an- alogues), parenteral nutrition, or nonoperative drainage procedures. 3. Grade C-Patients have severe clinical symptoms and signs of sepsis ororgan failure, or require reoperation for control of the fistula and its sequelae.

\section{Results}

\section{Surgical procedures}

Patient characteristics are shown in Table 1. From a total of 38 patients who were assessed for eligibility, 38 patients undergone pancreatectomy (PD [Group 1], $n=9$; Distal pancreatectomy [Group 2], $\mathrm{n}=29$ ) were retrospectively reviewed in a prospectively collected database. Short-term outcome in terms of procedure-related complication was evaluated. There was no significant difference in demographic features in both groups (Table 1). Majority of patients was female (Group 1: 66.6\%, Group 2: 75.8\%) and had no significant comorbidity (Group 1: 88.8\%, Group 2: 82.4\%). Patients in group 1 rarely presented with obstructive jaundice (median bilirubin level = $11.1 \mathrm{umol} / \mathrm{L}$ ).

Table 1: Demographic and clinical characteristics of PD vs. DP.

\begin{tabular}{|l|l|l|l|}
\hline Characteristics & $\begin{array}{l}\text { PD } \\
(\mathbf{n = 9 )}\end{array}$ & $\begin{array}{l}\text { DP } \\
(\mathbf{n}=\mathbf{2 9})\end{array}$ & $\boldsymbol{p}$ value \\
\hline Age (yr) & 46.00 & 53.00 & 0.436 \\
\hline Sex (M:F) & $3: 6$ & $7: 22$ & 0.909 \\
\hline comorbidity & $1(11.1 \%)$ & $5(17.2 \%)$ & 1.000 \\
\hline Tbil & 11.10 & 8.10 & 0.636 \\
\hline Alb & 42.00 & 42.30 & 0.543 \\
\hline CEA & 1.20 & 1.00 & 1.000 \\
\hline CA199 & 10.05 & 10.60 & 0.723 \\
\hline
\end{tabular}

PD: Pancreatico Duodenectomy; DP: Distal Pancreatectomy.

Table 2: Intraoperative data and post-operative morbidity.

\begin{tabular}{|c|c|c|c|}
\hline Characteristics & $\begin{array}{l}P D \\
(n=9)\end{array}$ & $\begin{array}{l}D P \\
(n=29)\end{array}$ & $p$ value \\
\hline Operation duration & 470.00 & 287.00 & $<0.001$ \\
\hline Length of stay (day) & 20.00 & 14.00 & 0.032 \\
\hline Blood loss (ml) & 600.00 & 350.00 & 0.013 \\
\hline $\begin{array}{l}\text { Pathology } \\
\text { Benign } \\
\text { Carcinoma }\end{array}$ & $\begin{array}{l}9(100 \%) \\
0(0.0 \%)\end{array}$ & $\begin{array}{l}19 \text { (65.5\%) } \\
10(34.5 \%)\end{array}$ & 0.105 \\
\hline $\begin{array}{l}\text { Pancreatic fistula } \\
\text { No } \\
\text { A } \\
\text { B } \\
\text { C }\end{array}$ & $\begin{array}{l}5 \\
3 \\
0 \\
1\end{array}$ & $\begin{array}{l}3 \\
19 \\
7 \\
0\end{array}$ & 0.004 \\
\hline Complication & $3(33.3 \%)$ & $3(10.3 \%)$ & 0.721 \\
\hline $\begin{array}{l}\text { Complication grade } \\
\text { No } \\
\text { III }\end{array}$ & $\begin{array}{l}6 \\
3\end{array}$ & $\begin{array}{l}26 \\
3\end{array}$ & 0.259 \\
\hline
\end{tabular}

PD: Pancreatico Duodenectomy; DP: Distal Pancreatectomy. 
Citation: Xu B, Yuan X, Wong T, et al. (2020) Evaluation of Safety of Pancreaticoduodenectomy for Patients with Benign Conditions. Clin Hematol Res 3(1):38-41

\section{Short-term complications}

All patients underwent resection. The surgical data, complications among the two surgical procedure groups were compared (Table 2). Disease spectrum in group 1 included insulinoma $(n=1)$, pancreatic tuberculosis $(n=1)$, IgG4-related disease $(n=1)$, mucinous cystadenoma $(n=1)$, splenic aneurysm $(n=1)$, serous cystadenoma $(n=2)$ and benign neuroendocrine tumor $(n=2)$. Group 1 had significantly longer operation duration (470 min vs. $287 \mathrm{~min}, \mathrm{p}<0.001$ ) (Table 2), longer hospital stay (20 days vs. 14 days, $p=0.032$ ) and more interoperative blood loss ( $600 \mathrm{ml} v \mathrm{ss} .350 \mathrm{ml}, \mathrm{p}=0.013$ ) than group 2. The postoperative pancreatic fistula rate of Group 1 was significantly lower than Group 2 (Grade A: $33.3 \%$ vs. $65.5 \%$; Grade B: $0 \%$ vs. $24.1 \%$; Grade C: $11.1 \%$ vs. $0 \%$ ), ( $p=$ 0.004). According to the Clavien-Dindo classification of surgical complications [6], there was no significant difference in other severe postoperative complications between the two groups. There was also no hospital mortality in both groups.

\section{Discussion}

There is only limited data on the incidence or prevalence of pancreatic cysts in the general population. The incidence of detected pancreatic cysts has been reported to range from as low as $0.7 \%$ to as high as $36.7 \%[2,7,8]$. The short-term complications associated with PD have been extensively studied. However, PD is an exceedingly rare procedure in benign pancreatic conditions. The current study presents a series of patients with benign pancreatic head disease who underwent tumor resection at one institution over 5-years. Perioperative factors and postoperative complications were further compared among the PD, and DP groups.

The PD is gold standard treatment for benign pancreatic head disease that contains insulinoma, mucinous cystic neoplasm, pancreatic tuberculosis, splenic aneurysm, serous cystadenoma, macrocystic serous cystadenoma, IgG4-related autoimmune pancreatitis in this study. The insulinoma and splenic aneurysm can cause severe symptoms. The mucinous cystic neoplasm, serous cystadenoma and macrocystic serous cystadenoma are precancerous lesions with progress to cancer [1,2,8-10]. The pancreatic tuberculosis and IgG4-related disease are very rare, and can be cured by non-surgical methods, if it can be diagnosed. However, diagnosis is very difficult [11].

Pancreatic benign tumors progress to pancreatic cancer due to atypical early symptoms of pancreatic disease, only 15 to 20 percent of patients are candidates for pancreatectomy. The prognosis of pancreatic cancer is poor even in those with potentially resectable disease, and despite progress in surgical techniques and adjuvant therapy, the evidence that outcomes are improving over time is equivocal. However, in a study of the National Cancer Database, among those who were candidates for resection of pancreatic cancer, 38 percent were not offered surgery $[3,12]$. PD is a multiorgan resection requiring tissue loss of the stomach, extrahepatic biliary structures, and duodenum [13].

Underutilization of surgery for early-stage pancreatic cancer may be related to socioeconomic factors as well as physician pessimism regarding the prognosis of the disease. Therefore, it is necessary to evaluate the safety of pancreatic head benign disease to resection, reduce patient and doctor concern, increases tumor resection and improves patient prognosis.

It is well established that resection of the pancreas is not without a high risk of complications, and Erika, et al. reported that $40 \%-50 \%$ of patients experienced short-term complications after PD [14]. In the current series, the morbidity was $1 / 3$. There was no statistically significant difference among the two groups, but pancreatic fistula was more likely to occur after DP than after PD. Studies have shown that pancreatic fistula is generally seen more often in patients with a soft pancreas and small pancreatic duct [15]. Therefore, the patients with benign disease of the pancreas underwent PD in this study were at lower risk than DP group for pancreatic fistula.

To conclude, in this study, we found that no mortality and acceptable morbidity, and PD can be safely and is beneficial to patients with benign pancreatic head diseases if there is no compromise of oncologic radicality.

\section{Acknowledgements}

\section{Funding sources}

This research did not receive any specific grant from funding agencies in the public, commercial, or not-for-profit sectors.

\section{Conflict of Interest}

None of the authors has any source of financial or other support, or any financial of professional relationship that may pose a competing interest.

\section{References}

1. De Jong K, Nio CY, Hermans JJ, et al. (2010) High prevalence of pancreatic cysts detected by screening magnetic resonance imaging examinations. Clin Gastroenterol Hepatol 8: 806-811.

2. Laffan TA, Horton KM, Klein AP, et al. (2008) Prevalence of unsuspected pancreatic cysts on MDCT. AJR Am J Roentgenol 191: 802-807.

3. Bilimoria KY BD, Ko CY, Stewart AK, et al. (2007) National failure to operate on early stage pancreatic cancer. Ann Surg 246: 173-180.

4. Allen PJ, Kuk D, Castillo CF, et al. (2017) Multi-institutional validation study of the American joint commission on cancer (8th Edition) changes for $\mathrm{T}$ and $\mathrm{N}$ staging in patients with pancreatic adenocarcinoma. Ann Surg 265: 185-191.

5. Shrikhande SV, Sivasanker M, Vollmer CM, et al. (2017) Pancreatic anastomosis after pancreatoduodenectomy: A position statement by the International Study Group of Pancreatic Surgery (ISGPS). Surgery 161: 1221-1234.

6. Dindo D, Demartines N, Clavien PA (2004) Classification of surgical complications: A new proposal with evaluation in a cohort of 6336 patients and results of a survey. Ann Surg 240: 205-213.

7. Fernandez Del Castillo C, Targarona J, Thayer SP, et al. (2003) Incidental pancreatic cysts: Clinicopathologic characteristics and comparison with symptomatic patients. Arch Surg 138: 427-433. 
8. Spinelli KS, Fromwiller TE, Daniel RA, et al. (2004) Cystic pancreatic neoplasms: observe or operate. Ann Surg 239: 657-659.

9. Kim AL, Choi YI (2017) Safety of duodenal ampullectomy for benign periampullary tumors. Ann Hepatobiliary Pancreat Surg 21: 146-150.

10. Lee CS, Kim EY, You YK, et al. (2018) Perioperative outcomes of laparoscopic pancreaticoduodenectomy for benign and borderline malignant periampullary disease compared to open pancreaticoduodenectomy. Langenbecks Arch Surg 403: 591-597.

11. Phil A Hart, Yoh Zen, Suresh T Chari (2015) Recent advances in autoimmune pancreatitis. Gastroenterology 149: 39-51.

12. Timothy R Donahue, Howard A Reber (2015) Surgical management of pancreatic cancer-pancreaticoduodenectomy. Seminars in Oncology 42: 92-109.
13. Beger HG, Mayer B, Poch B (2018) Parenchyma-sparing, local pancreatic head resection for premalignant and low-malignant neoplasms-A systematic review and meta-analysis. Am J Surg 216: 1182-1191.

14. Lindholm EB, Alkattan AK, Abramson SJ, et al. (2017) Pancreaticoduodenectomy for pediatric and adolescent pancreatic malignancy: A single-center retrospective analysis. J Pediatr Surg 52: 299-303.

15. You DD, Paik KY, Park IY, et al. (2019) Randomized controlled study of the effect of octreotide on pancreatic exocrine secretion and pancreatic fistula after pancreatoduodenectomy. Asian J Surg 42: 458-463. 